\title{
Dissipation in hysteretic rubber mount models
}

\author{
R. Penas \\ Groupe PSA, Vélizy-Villacoublay, France \\ Laboratoire PIMM, Arts et Metiers ParisTech, CNRS, CNAM, HESAM, Paris, France
}

A. Gaudin

Groupe PSA, Vélizy-Villacoublay, France

A. Kreis

Groupe PSA, Poissy, France

E. Balmes

Laboratoire PIMM, Arts et Metiers ParisTech, CNRS, CNAM, HESAM, Paris, France

SDTools, Paris, France

\begin{abstract}
Rubber mounts are elements of extreme importance in automotive suspension, and accurate modeling is crucial for comfort design. While mount characterization is typically done using cycles, actual performance is often associated with transients. The paper thus focuses on the impact of power dissipation on suspension models during transients. For scalar or 0D hysteretic models that respect Madelung rules and Masing's law, a method is introduced to compute instant dissipation even for models where it is not explicitly available. It is shown that stiffness and dissipation depend only on the last turning point and this dependence should be the core aspect for identifying and modeling hysteretic dissipation. The second section introduces two different 0D models having the same full cycle dissipation and force amplitude, thus the same storage and loss moduli in a first harmonic approximation of the mount behavior, though having different instantaneous dissipation. The case of a transient starting torque soliciting a suspended powertrain is finally considered. The different suspension models are shown to have different instant dissipation which might deeply modify the conclusions drawn from the dynamic simulation.
\end{abstract}

\section{INTRODUCTION}

Rubber mounts are widely used in automotive industry due to their unique combination of moderate stiffness and high dissipation. These properties are particularly interesting for softening and filtering vibrations in articulations between moving parts. Despite the intense utilization of rubbery materials, their behavior is still subject of active research, given that current modeling strategies cannot provide fully predictive design.

One of the main modeling demands for this type of articulations comes from multibody simulations where a low computational cost is a modeling constraint. For this reason, scalar or 0D models are the most commonly used for emulating rubber articulations. These models can describe and accumulate different nonlinear effects, while keeping a relatively low complexity. Models of this kind are often found in the specific literature such as Coveney \& Johnson
(2000), Sjöberg (2002), Bourgeteau (2009).

To identify this complex behavior, the most used technique is to impose cyclic solicitations and fit the force response with scalar models. Cyclic identification does not provide information on dissipation during transients. The first section describes hysteretic models which are the focus of this work. It is in particular shown how instant dissipation, even if is not accessible experimentally, can be computed and used as a valuable asset for analysis, by giving an estimation of when and where the dissipation is concentrated. The second section describes two models equivalent from the point of view of cyclic dissipation but leading to very different behavior in an engine start simulation. 


\section{HYSTERETIC MODEL}

In the scope of this work, hysteresis is taken as a rate-independent behavior that respects the Madelung rules and the Masing's law (see Brokate \& Sprekels (2012)). The rate-independence means that viscoelastic contribution is not taken in account and should be object of further work.

The Madelung rules state that :

- Any curve $\Gamma_{1}$ emanating from a turning point $A$ of the force/displacement graph (figure 1) is uniquely determined by the coordinates of $A$.

- If any point $B$ on the curve $\Gamma_{1}$ becomes a new turning point, then the curve $\Gamma_{2}$ originating at $B$ leads back to the point $A$.

- If the curve $\Gamma_{1}$ is continued beyond the point $A$, then it coincides with the continuation of the curve $\Gamma$ which led to the point $A$ before the $\Gamma_{1}-\Gamma_{2}$ cycle was traversed.

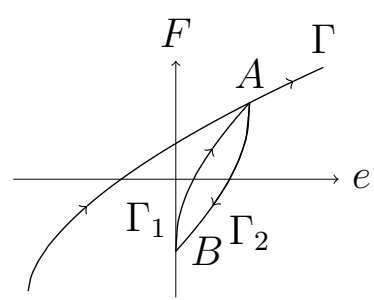

Figure 1: Illustration of Madelung rules

Noting + the curve that leads $A$ to $B$ and - the curve that leads $B$ to $A$. The cycle closure, or the fact that every turning point must be passed though again before reaching greater deformation, can be written as the integral of the tangent stiffness or slope $K(e)=$ $\partial f / \partial e$ over two half cycles

$$
\begin{aligned}
0 & =\int_{e_{A}}^{e_{B}} K_{+}\left(e-e_{A}\right) d e+\int_{e_{B}}^{e_{A}} K_{-}\left(e-e_{B}\right) d e \\
& =\int_{0}^{e_{B}-e_{A}} K_{+}(e) d e+\int_{e_{B}-e_{A}}^{0} K_{-}(e) d e \\
& =\int_{0}^{e_{B}^{-e_{A}}}\left(K_{+}(e)-K_{-}(-e)\right) d e
\end{aligned}
$$

Since this is true for any cycle length, the tangent stiffness is an even function of the displacement, that is $K_{+}(e)=K_{-}(-e)$. Thus in figure 2 rather than displaying the classical cycle on the top, one chooses to represent the tangent stiffness.

Other hysteretic model main characteristic is the fact that the first loading curve should be homothetic to the first unloading curve, with a scale factor of two, known as Masing's law (Brokate \& Sprekels 2012). Combining both laws, illustrated in figure 2, one can represent $K(e)$ as a function of distance to turning point with a factor 2 on $e$ when starting from 0 .

Concerning dissipation, the parity property is quite useful, since dissipation can be split equally between

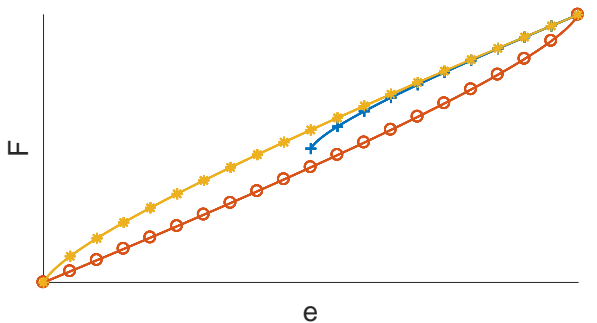

a)

b)

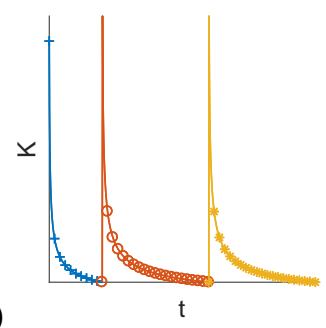

c)

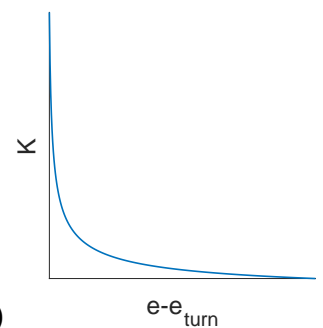

Figure 2: Illustration of Masing's law. a) classical cycle, tangent stiffness as function time (b) and distance to turning point (c)

loading and unloading. From any turning point $A$ and current point $B$, it is possible to close a cycle going back to point $A$, where dissipation verifies

$$
\begin{aligned}
E_{d} & =\int_{e_{A}}^{e_{B}} F_{d i s} d e=\frac{1}{2} \oint F d e \\
& =\frac{1}{2}\left(\int_{e_{A}}^{e_{B}} F_{+} d e+\int_{e_{B}}^{e_{A}} F_{-} d e\right) \\
& =\int_{e_{A}}^{e_{B}} F(e) d e-F\left(e_{B}-e_{A}\right)\left(e_{B}-e_{A}\right)
\end{aligned}
$$

which can be used to compute the instantaneous value of dissipated energy.

These rules are applicable when neglecting viscoelastic and nonlinear elastic effect. Figure 3 illustrates tests with triangular enforced displacement. A low, constant speed was used to minimize viscoelastic effects which should remain negligible. Nonlinear elastic effects, from both geometric and material natures are also negligible due to the use of deformations below $10 \%$.
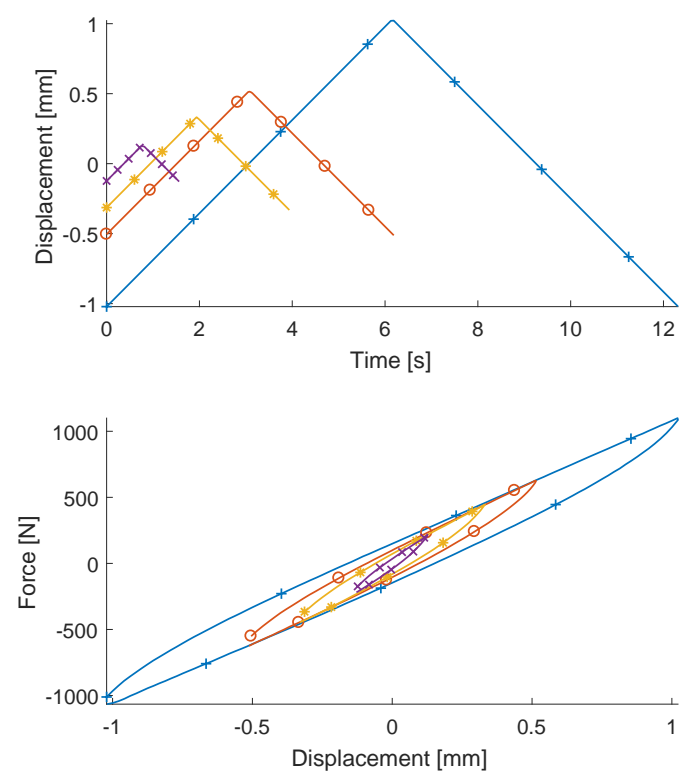

Figure 3: Imposed displacement and force-displacement response 
The results are shown in the force/displacement plane in figure 3. This plot suggests that the stated hypotheses are plausible, but a simpler verification is to show, as in figure 4, the instant stiffness as function of the distance to the last turning point. In this representation, the verification of hypotheses is confirmed by the superposition of all curves.

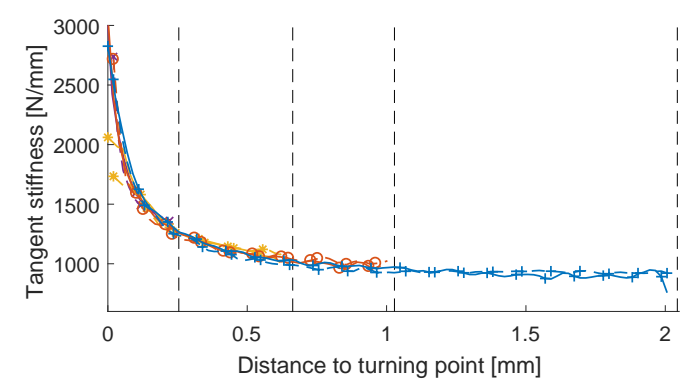

Figure 4: Tangent stiffness as function of distance to last turning point

Since the $K\left(e-e_{\text {turn }}\right)$ curve completely defines an hysteretic model, a non-parametric representation of the behavior is simply achieved though its discretization. Segalman (2005) in his work proposes a model based on a rheologic representation, shown in figure 5 . This model allows the reproduction of the curve respecting both laws with a continuous force evolution and control on the accuracy.

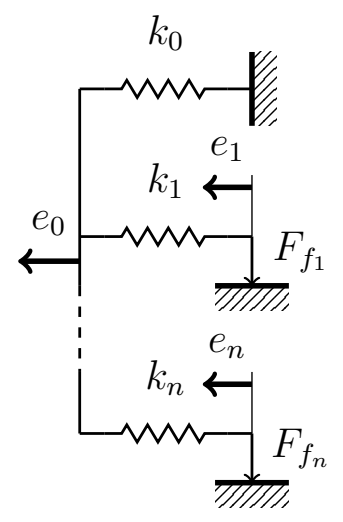

Figure 5: Rheologic scheme for Iwan's model

Despite needing a high number of Jenkins cells for accurate representation, as seen on figure 6 , the low computational effort associated to each cell makes it an interesting model for application purposes.

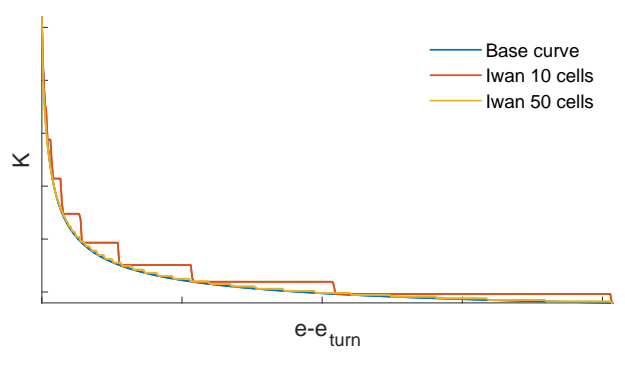

Figure 6: Illustration of Iwan's model response

An alternate hysteretic model that respects the stated hypotheses is the STS model, introduced and detailed by Coveney \& Johnson (2000). This model is based on an spring in parallel of an assembly of identical Jenkins cells in series, shown in figure 7. By making the springs stiffness tend to infinity and the friction forces tend to zero, with their product constant $\left(k_{t} F_{f}=C_{t}\right)$, one reaches to a continuous model, defined by the equation 3 .

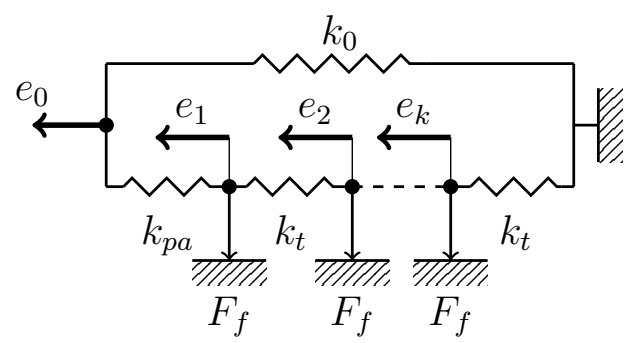

Figure 7: Rheologic scheme for the STS model

$$
\begin{aligned}
F-F_{\text {turn }}= & \sqrt{N C_{t}\left|e-e_{t u r n}\right|+\frac{N C_{t}}{2 k_{p a}}} \\
& -\frac{N C_{t}}{2 k_{p a}} \operatorname{sign}\left(e-e_{\text {turn }}\right)
\end{aligned}
$$

\section{NONLINEAR ELASTICITY AND HYSTERETIC DAMPING}

In order to apply the the models on section 2, identification tests were made on three mounts that compose a powertrain suspension. These tests were made with enforced displacement at constant and low speed, and the results are illustrated by figure 8 .

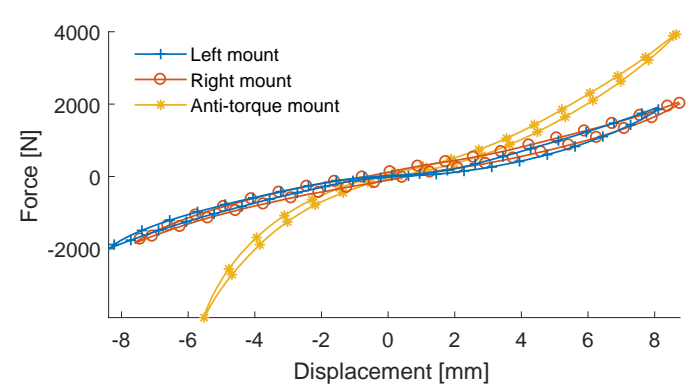

Figure 8: Force/displacement response for the three tested mounts

The curly shape of test data suggests the presence of a non linear elastic component and classical dissipative models are not capable of representing this kind of behavior. A nonlinear elastic model was built using the mean test load for each displacement and is shown in figure 9 left.

The remaining forces, shown in figure 9 right, are supposed to be the generated by the dissipative model in parallel of the nonlinear elastic model. The left mount (and on a smaller scale the anti torque mount) presents significantly less dissipation around null displacement. It is probably because a significant part of the rubber is not excited within small displacements, and after a certain level, it is solicited by a self-contact. 

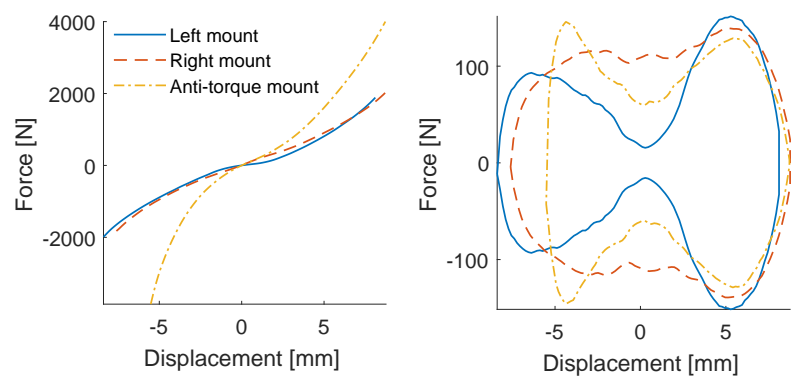

Figure 9: Non-linear elastic response (left) and dissipative forces (right) for the tested mounts

Tracing the stiffness as function of distance to the turning point without the nonlinear elastic contribution, in figure 10, as it was suggested in section 2, one can see that its evolution for small distances to the turning point is quite similar to the ones provided by hysteretic models. The absence of dissipation around null displacement creates bumps in the curves between 6 and $10 \mathrm{~mm}$, more visible for the left mount. The end of the curve shows strong negative stiffness, as for a form of symmetry, which is probably due to the removal of a nonlinear elastic part.

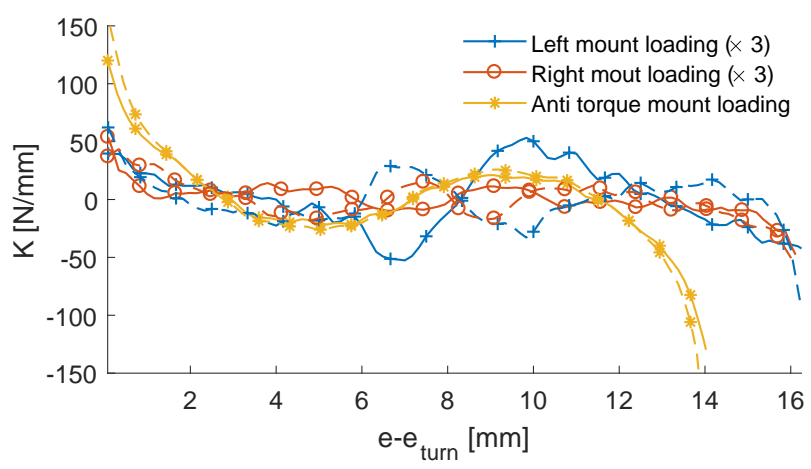

Figure 10: Stiffness in function of the distance to the last turning point without nonlinear elastic component

To fit the dissipation in parallel of the non-linear elasticity, two different models were used: one is the STS model and the other is a simple viscous dissipation. Both models were tuned to dissipate the same amount of energy for a harmonic solicitation with frequency close to the powertrain rolling frequency (around $10 \mathrm{~Hz}$ ), with the same amplitude as the identification tests. Figure 11 shows the instant dissipation for both models of the right mount under the stated load, and even if the total dissipation is the same, their instant dissipation is different.

\section{APPLICATION CASE}

With the mounts identified, a model of the whole powertrain suspension was developed. The powertrain unit modeled by its mass and inertia matrix, while the three mounts are attached on one side to the powertrain unit, and the other to the chassis, considered as rigid as shown in figure 12. The model input is the engine torque, applied in the direction of the

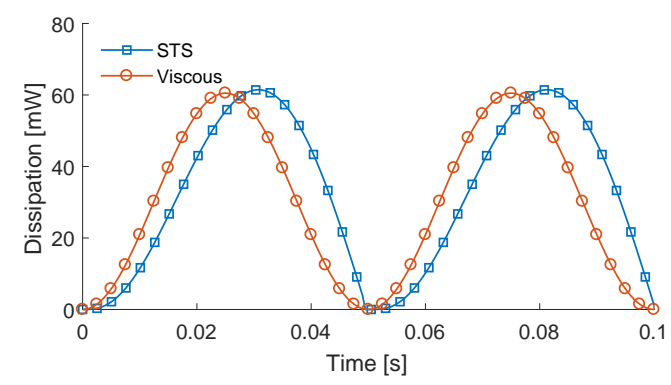

Figure 11: Instantaneous dissipation for a STS model and a viscous model under harmonic solicitation

crankshaft and whose time evolution is illustrated in figure 13, and the outputs are the forces exerted by the three mounts on the body.

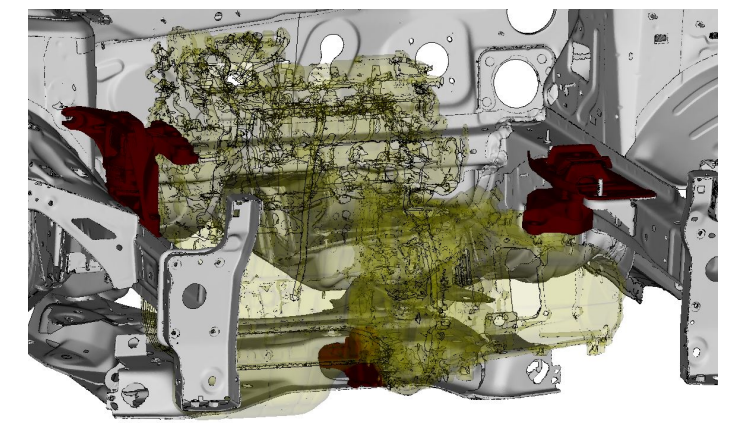

Figure 12: Assembled powertrain unit and its suspension

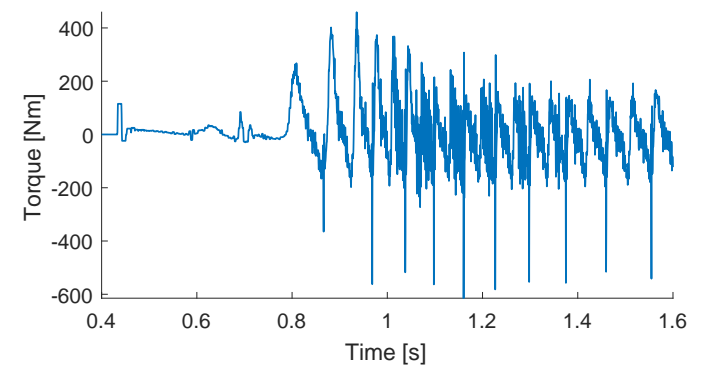

Figure 13: Measured torque for a starting engine

Figure 14 shows the experimental results and simulated forces for both models in the direction of the vehicle motion.

With forces and displacements available for post treatment, viscous dissipation may be explicitly computed, and hysteretic dissipation may be computed by equation 2 . Figure 15 illustrates the evolution of dissipated energy for both computed models, and it highlights the fact that for this transient simulation, the dissipated energy differs both in total dissipated energy and timing of when the energy is dissipated. This demonstrate that the original equivalence shown in 3 is not sufficient to provide a proper transient simulation. 

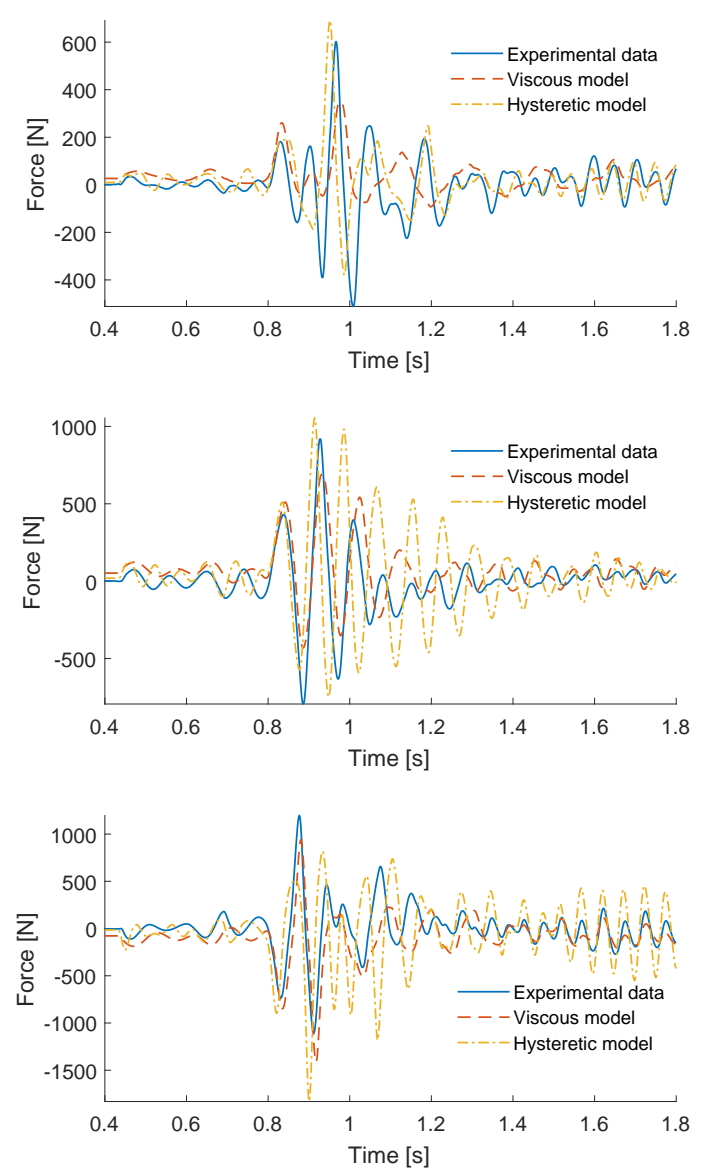

Figure 14: Measured and computed forces at the mounts. Top: Left mount; Middle: Right mount; Bottom: Anti-torque mount

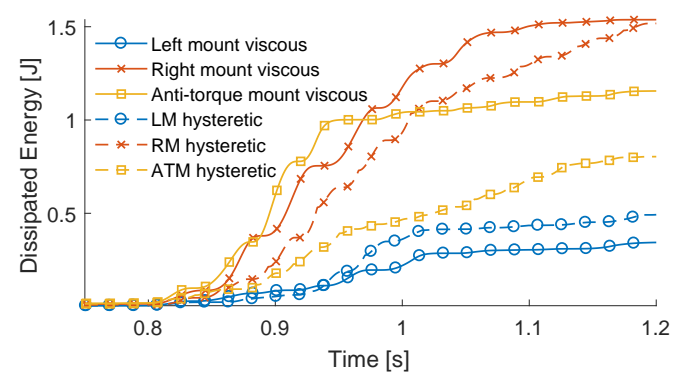

Figure 15: Dissipated energy for each model

To analyze the implications of this difference into the system level, a modal analysis was carried around the resting position of the engine. As expected, the six powertrain modes are three translational modes and three rotational modes, listed in table 1.

Table 1: Powertrain suspension modes

\begin{tabular}{ll}
\hline Mode description & Frequency \\
\hline Translation perpendicular to vehicle direction & $4.00 \mathrm{~Hz}$ \\
Translation in vehicle direction & $6.04 \mathrm{~Hz}$ \\
Translation in vertical direction & $7.13 \mathrm{~Hz}$ \\
Pitching powertrain & $9.18 \mathrm{~Hz}$ \\
Yawing powertrain & $11.66 \mathrm{~Hz}$ \\
Rolling powertrain & $13.38 \mathrm{~Hz}$ \\
\hline
\end{tabular}

Modal amplitudes are the decomposition of the displacement on the directions of the eigenvectors (Bianchi, Balmes, Vermot Des Roches, \& Bobillot 2010). This decomposition of the displacement vec- tor $q$ is given by

$q_{j}=\phi_{j}{ }^{T} M\{q\}$

with $\phi$ the mass normalized eigenvectors and $M$ the mass matrix, and $q_{j}$ the amplitude associated to the mode $j$. The time derivative of those amplitudes yield the modal speeds which are also necessary for computing modal energies using

$2 E_{j}=\dot{q}_{j}^{2}+\omega_{j}^{2} q_{j}^{2}$

with $E_{j}$ the energy and $\omega_{j}$ the frequency associated to mode $j$. This information is useful to know where the energy flows before being dissipated. Modal energy evolution through time is shown in figure 16, for both hysteretic and viscous models.
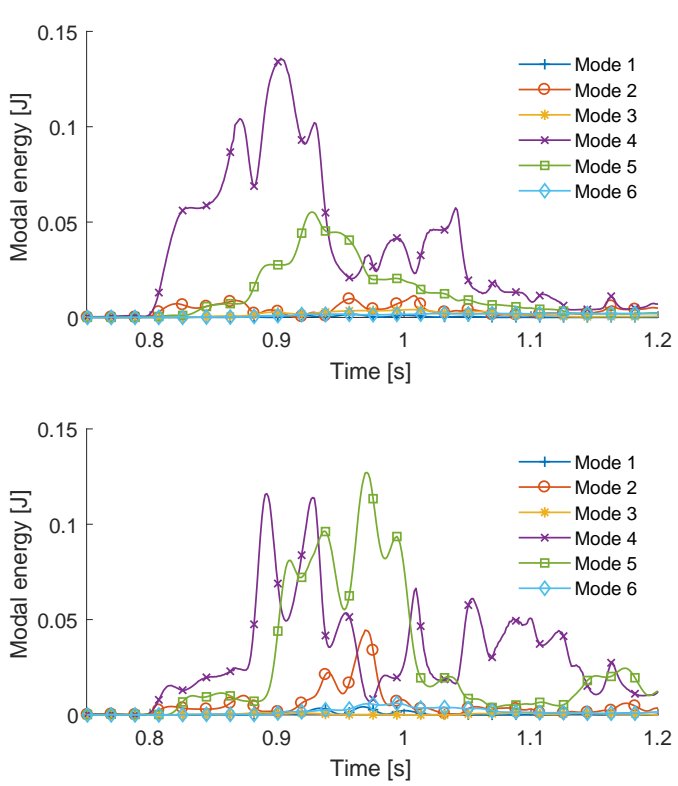

Figure 16: Modal energies for each model. Top: Viscous model; Bottom: Hysteretic model

Modes 4 and 5 take most of the imposed energy on the system, which is natural, since they represent the rotations along the direction of the imposed torque. The different dissipation models impact strongly on how the energy is transmitted to modes, leading to conflicting information of which mode is more important to address.

\section{CONCLUSION}

This work focused on a specific range of models whose objective is to emulate the rate-independent nonlinear component of rubber mounts behavior. Models of this type used in industry are often identified with full-cycle dissipation equivalence. This equivalence does not take into account all the necessary aspects for a predictive transient simulation. To sustain this argument, an application case where a starting powertrain unit was simulated with two suspension models that differ only on the dissipation instants and the simulations would lead to different design choices. 
The need for identification strategies accounting for a conservative nonlinear elastic part, rate-dependent dissipation (viscoelastic) and rate-independent (hysteretic) dissipation has only been illustrated here and should be an important aspect of future work. For industrial application, evolution of dissipation with non-linear elasticity may also represent an important component of the model for many mounts.

\section{REFERENCES}

Bianchi, J. P., E. Balmes, G. Vermot Des Roches, \& A. Bobillot (2010). Using modal damping for full model transient analysis. application to pantograph/catenary vibration. In ISMA, pp. 376.

Bourgeteau, B. (2009). Modélisation numérique des articulations en caoutchouc de la liaison au sol automobile en simulation multi-corps transitoire.

Brokate, M. \& J. Sprekels (2012). Hysteresis and phase transitions. Springer.

Coveney, V. A. \& D. E. Johnson (2000). Rate-dependent modeling of a highly filled vulcanizate. Rubber chemistry and technology 73, 565-577.

Segalman, D. J. (2005). A four-parameter iwan model for laptype joints. Journal of Applied Mechanics 72(5), 752-760.

Sjöberg, M. (2002). On dynamic properties of rubber isolators. 